\section{BOOK REVIEW}

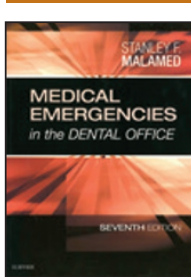

MEDICAL EMERGENCIES IN THE DENTAL OFFICE, 7TH EDITION

S. F. Malamed

Elsevier

price $€ 62.99 ;$ pp 576

ISBN 9780323171229

The seventh edition of Medical emergencies in the dental office is a glossy paperback designed as an authoritative resource for all the medical emergencies likely to occur. This 550 page colourful and aesthetically pleasing text is methodically written and well structured. This text is written by Stanley F Malamed (DDS) who is a Dentist Anesthesiologist in America. Potential readers must therefore be aware this is written from an American perspective and does not comply with Resuscitation Council guidelines (UK).

The book is split into eight parts further divided into 31 comprehensive chapters and covers a very extensive list of subjects. Some subjects the reader may be surprised to find in a medical emergencies text, for example, chapter four, Legal considerations.

Despite its somewhat daunting size, it is very easy to read and provides a very comprehensive reference for a variety of medical emergencies. Each medical emergency is given a very detailed background of risk factors, pathophysiology, prevention and clinical manifestations before management of the condition is discussed.

The topics covered range from simple syncope through to foreign body airway obstruction and cardiac arrest. Readers may be surprised to find acute adrenal insufficiency and thyroid gland dysfunction covered. These chapters are extremely interesting but may be of little practical use to the majority of general dental practitioners.

As mentioned, this book is written from an American perspective and with this in mind, readers should show caution in following protocols that do not comply with UK guidelines. For example, basic life support in this text recommends a very different approach to that of the Resuscitation Councils 'ABCDE'.

In conclusion, this book is comprehensive in its discussion and management of medical emergencies. However, given it does not comply with UK guidelines I could not recommend this text alone to anyone wishing to broaden their knowledge and experience. It is however an extremely interesting read and an excellent foundation for any undergraduate dental student.

\section{S. MCKERNON}

\section{HAVE YOUR SAY ON CPD}

Do you think CPD is valuable to you? Are you interested in having your say on CPD? The you will be glad to know UCL Eastman are carrying out a nationwide survey to find out your views on the current state of CPD.

If you have an opinion and wish to complete the online survey, visit https://www.surveymonkey. com/s/7YD9T9S.

\title{
WHICH? REPORT FINDS ONE IN THREE PRACTICES 'REFUSING NEW PATIENTS'
}

Consumer group Which? has discovered one in three NHS-listed dentists in England is refusing to take on any new NHS patients.

The report also identified significant waiting lists in certain areas of the country, with some people having to wait up to nine months for treatment.

The Which? study is based on findings from undercover researchers who contacted 500 dental surgeries listed on the official NHS Choices website, posing as new NHS patients.

They found the website's information was often inaccurate, with three in ten surgeries turning them down for appointments.

As well as some not having availability, a further three in ten of those that could take on new NHS patients had waits of at least two weeks for an appointment, with one saying it would be eight to nine months before the patient could be seen.

Some practices also made people 'jump through unnecessary hoops' such as visiting the surgery to fill out forms or asking patients to pay deposits.

In response to report, The British Dental Association (BDA) has consistently called for action on the comprehensively flawed payments system at the heart of NHS dentistry.

Since April 2006, NHS dentists in England and Wales have been paid according to how many Units of Dental Activity (UDA) they do in a year. This system has proved a serious obstacle to a preventive approach to dentistry, by rewarding dentists for completed treatment on the basis of a 'points' system, not the number of items in the treatment plan or net improvements in oral health.

The UDA system has fuelled waiting lists, with practices contractually obliged not to exceed a fixed number of 'points' in a given year. Mick Armstrong, Chair of the British Dental Association, said: 'The government remains committed to a byzantine system that has failed both dentists and their patients. These targets might suit a Whitehall spreadsheet but do not reflect the reality of millions of people receiving and seeking NHS treatment.

'From day one these arbitrary targets have proved a real obstacle for new NHS patients. Dentists often have more patients lining up than they have UDAs, but the UDA must come first. Successive governments appear to have been more attached to acronyms than to patients seeking care.

'When dentists 'over perform' and treat more NHS patients than they are allocated, that's not 'success', and they pay for it out of their own pockets. Many would like to see more patients, but this is impossible within rigid contracts.

'Money being clawed back from dentistry is not being reinvested in dentistry. We have a shrinking budget that only covers 56 per cent of the population. For far too long oral health has been left out of the health debate, and this new evidence provides fresh impetus for government to reassess its agenda.

'Two watered-down versions of the UDA are now going into trials as a possible basis for a new dental contract. We want to see prevention at the heart of dentistry. We want real and agreed clarity for both patients and practitioners on what the NHS will cover, and at what cost. None of that will be possible until the UDA is consigned to the dustbin of history.'

The report is the latest in a series by Which? who earlier this year claimed some dentists aren't being upfront about payment costs or what treatment patients are entitled to on the NHS.

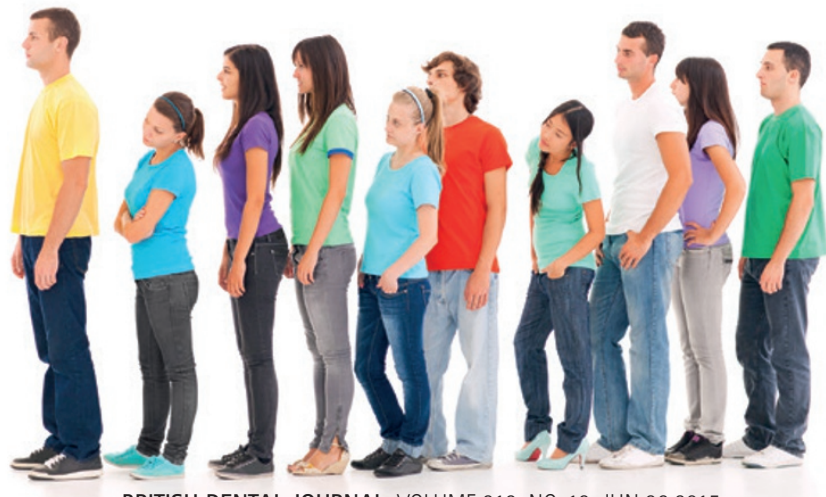

BRITISH DENTAL JOURNAL VOLUME 218 N0. 12 JUN 262015 